\title{
A population-based study of syphilis and sexually transmitted disease syndromes in north-western Tanzania. 2. Risk factors and health seeking behaviour
}

\author{
J Newell, K Senkoro, F Mosha, H Grosskurth, A Nicoll, L Barongo, M Borgdorff, \\ A Klokke, J Changalucha, J Killewo, J Velema, A S Muller, J Rugemalila, D Mabey, \\ R Hayes
}

\begin{abstract}
Objective-To determine risk factors for syphilis and sexually transmitted disease (STD) syndromes, and to study health seeking behaviour among those with STD syndromes, in the population of Mwanza Region, North-Western Tanzania.

Methods-A population-based random cluster sample survey, stratified by rural, roadside or urban residence, of 4173 individuals aged 15-54 years was performed in 1990-91. The seroprevalence of syphilis and the prevalence and incidence of self-reported genital ulcer syndrome (GUS) and genital discharge syndrome (GDS) are reported in the accompanying paper. This paper reports on risk factors for these conditions and on health seeking behaviour among those reporting them.
\end{abstract}

Results-In both sexes, the risk of STDs increased with the reported number of sexual partners in the previous five years. Men who were separated, divorced or widowed were at increased risk of STDs, but this was not the case among women. Higher educational status was associated with an increased risk of urethral discharge in males but with a decreased prevalence of syphilis in females. Male circumcision was associated with an increased risk of urethral discharge but a reduced prevalence of syphilis. Nearly all men, and $90 \%$ of women, reporting symptoms of genital discharge or ulceration had sought treatment. Of these, approximately $70 \%$ of males and $60 \%$ of females had sought treatment in the official health sector.

Conclusions-Targetted health education concerning risk reduction for HIV infection and other STDs should be a high priority in this population. Improved case management of STDs in health centres and dispensaries may have a substantial impact on the incidence of these infections.

(Genitourin Med 1993;69:421-426)

Introduction

Sexually transmitted diseases (STDs) have been a major public health problem in developing countries for many years, but their importance has been recognised increasingly since the onset of the HIV/AIDS epidemic. ${ }^{1-3}$ While several studies have investigated risk factors for HIV infection in Africa, little is known about risk factors for other STDs, and there have been few population-based studies of these diseases.

While in many cases risk factors for HIV infection and other STDs are likely to be similar, there are several reasons why they may differ. Firstly the bacterial STDs, unlike HIV infection, are treatable. The availability of treatment will therefore influence the prevalence and incidence of these diseases. Secondly, STD pathogens differ in their transmissibility; risk factors for an infection with a high probability of transmission after a single contact may not be the same as those for a less easily transmitted infection. Thirdly, HIV infection has been circulating in human populations for a much shorter time than other STDs, and so is more likely to be concentrated in urban and highly mobile populations.

Nevertheless the identification of risk factors for other STDs has important implications for the targetting of HIV control programmes. In rural populations which currently have a low HIV prevalence, risk factors for the "classical" STDs are likely to prove risk factors for HIV infection once it becomes established. Furthermore, recent evidence indicates that such STDs may facilitate the heterosexual transmission of HIV, ${ }^{4-6}$ suggesting that interventions to reduce STD prevalence may be effective in restricting the spread of HIV.

In view of the serious sequelae of the bacterial STDs, ${ }^{7}$ particularly among women and infants, and their possible role in HIV transmission, there is an urgent need to improve the management of these infections at all levels of the health services of developing countries. However, it is likely that in Africa many individuals with symptomatic STDs seek treatment outside the official health sector. There is little published information on the health seeking behaviour of people with STDs in African populations.

We have conducted a region-wide population-based survey of HIV infection, syphilis and self-reported STD syndromes in Mwanza Region, North-Western Tanzania. The prevalence of and risk factors for HIV infection have been reported elsewhere. ${ }^{8}$ The prevalence and incidence of syphilis and STD 
syndromes are reported in the accompanying paper. ${ }^{9}$ We report here risk factors for syphilis and STD syndromes, and the health-seeking behaviour of those reporting these syndromes.

\section{Methods}

The geography and demography of Mwanza Region, and the selection of study subjects are described in the accompanying paper. ${ }^{9}$ Briefly, 5145 adults aged 15-54 years were selected by random cluster sampling from the regional population, in three strata: urban (Mwanza Municipality: 1554 selected), roadside (1157) and rural (2434). Of those selected, the numbers participating were 1187,962 and 2024 respectively, giving coverage rates of $76 \%, 83 \%$ and $83 \%$.

Respondents were interviewed confidentially using a structured questionnaire. In addition to age, sex and residence, and symptoms of genital discharge or ulceration (see accompanying paper $^{9}$ for the wording of these questions), information was collected on possible risk factors, including: level of education, occupation, marital status; number of sexual partners in the past five years, travel to Mwanza town or out of the region in the past two years and, for males, circumcision status. Those who reported that they had ever had genital discharge or ulceration were asked whether they had sought treatment and, if so, where.

Respondents also gave a sample of venous blood for serological testing for HIV and syphilis, using laboratory methods as described in the accompanying paper. ${ }^{9}$

Risk factor analyses were conducted for (i) active syphilis (positive RPR and TPHA tests); (ii) reported GDS during the past year; and (iii) reported GUS during the past year. Separate analyses were conducted for males and females, since risk factors may differ between the sexes. Two estimates of the odds ratio (OR) are presented for each risk factor: the Mantel-Haenszel OR, adjusted only for age group and residential stratum; and the OR (and its 95\% confidence interval (CI)) obtained by logistic regression, adjusted additionally for confounding factors selected because they had an appreciable effect on the regression parameters. The effects of the cluster sampling scheme were ignored in this analysis.

\section{Results}

Risk factors for syphilis

Overall prevalences of active syphilis (TPHA +, RPR +) were $8 \cdot 1 \%$ for males and $\mathbf{9 . 4 \%}$ for females, with lower prevalences in rural villages than in the urban and roadside strata. ${ }^{9}$ Active syphilis showed little variation with age or sex, except for a lower prevalence in men aged 15-24 than women of the same age in the urban and roadside strata $(5 \%$ vs $10 \% ; p=0.004)$. Prevalences of active syphilis by possible risk factors are shown in table 1, together with adjusted odds ratios. The following associations were identified by this analysis.

Active syphilis was less prevalent among women with four or more years of school education ( $\mathrm{OR}=0.6, \mathrm{CI}: 0.4-0.9)$, but there was no association with level of education among men. Compared with farmers, men conducting business or employed in office jobs or manual work $(\mathrm{OR}=0.6, \mathrm{CI}$ : $0.4-0.9$ ), or in other groups ( $\mathrm{OR}=0.2, \mathrm{CI}$ : $0 \cdot 0-0 \cdot 8)$, had significantly lower prevalences. Many of those in the third category were

Table 1 Risk factors for active syphilis (TPHA+, RPR+) in Mwanza Region

\begin{tabular}{|c|c|c|c|c|c|c|c|c|c|c|}
\hline \multirow[b]{2}{*}{ Risk factor } & \multicolumn{5}{|l|}{ MALES } & \multicolumn{5}{|l|}{ FEMALES } \\
\hline & $+v e / t o t$ & $\%$ & $O R^{*}$ & OR $¥$ & $(95 \% C I)$ & $+v e / t o t$ & $\%$ & $O R^{*}$ & OR $\ddagger$ & $(95 \% C I)$ \\
\hline $\begin{array}{l}\text { Education } \\
<4 \text { years } \\
\geq 4 \text { years }\end{array}$ & $\begin{array}{c}44 / 464 \\
126 / 1532\end{array}$ & $\begin{array}{l}9 \cdot 5 \\
8 \cdot 2\end{array}$ & $\begin{array}{l}1 \\
0.9\end{array}$ & $\begin{array}{l}1 \\
1 \cdot 0\end{array}$ & $\begin{array}{l}\mathrm{p}=0.90 \\
(0.6-1 \cdot 5)\end{array}$ & $\begin{array}{l}116 / 1002 \\
100 / 1155\end{array}$ & $\begin{array}{r}11 \cdot 6 \\
8 \cdot 7\end{array}$ & $\begin{array}{l}1 \\
0.6\end{array}$ & $\begin{array}{l}1 \\
0 \cdot 6\end{array}$ & $\begin{array}{l}\mathrm{p}<0.01 \\
(0.4-0.9)\end{array}$ \\
\hline $\begin{array}{l}\text { Occupation } \\
\text { Farmer } \\
\text { Emp'd/Busin's } \\
\text { Other }\end{array}$ & $\begin{array}{c}127 / 1339 \\
41 / 519 \\
2 / 137\end{array}$ & $\begin{array}{l}9 \cdot 5 \\
7.9 \\
1.5\end{array}$ & $\begin{array}{l}1 \\
0 \cdot 6 \\
0 \cdot 2\end{array}$ & $\begin{array}{l}1 \\
0 \cdot 6 \\
0 \cdot 2\end{array}$ & $\begin{array}{l}\mathrm{p}<0.01 \\
(0.4-0.9) \\
(0.0-0.8)\end{array}$ & $\begin{array}{c}201 / 1967 \\
14 / 139 \\
1 / 53\end{array}$ & $\begin{array}{r}10 \cdot 2 \\
10 \cdot 1 \\
1 \cdot 9\end{array}$ & $\begin{array}{l}1 \\
0 \cdot 8 \\
0 \cdot 2\end{array}$ & $\begin{array}{l}1 \\
0 \cdot 7 \\
0 \cdot 3\end{array}$ & $\begin{array}{l}0.2<\mathrm{p}<0.3 \\
(0 \cdot 4-1 \cdot 3) \\
(0 \cdot 0-2 \cdot 3)\end{array}$ \\
\hline $\begin{array}{l}\text { Marital status } \\
\text { Married } \\
\text { Single } \\
\text { Sep/Wid/Div }\end{array}$ & $\begin{array}{c}118 / 1186 \\
25 / 614 \\
27 / 179\end{array}$ & $\begin{array}{r}9 \cdot 9 \\
4 \cdot 1 \\
15 \cdot 1\end{array}$ & $\begin{array}{l}1 \\
0 \cdot 4 \\
1 \cdot 6\end{array}$ & $\begin{array}{l}1 \\
0 \cdot 5 \\
1 \cdot 7\end{array}$ & $\begin{array}{l}\mathrm{p}<0.005 \\
(0.3-0.9) \\
(1 \cdot 1-2 \cdot 7)\end{array}$ & $\begin{array}{c}143 / 1409 \\
25 / 379 \\
47 / 357\end{array}$ & $\begin{array}{r}10 \cdot 1 \\
6 \cdot 6 \\
13 \cdot 2\end{array}$ & $\begin{array}{l}1 \\
0 \cdot 6 \\
1 \cdot 3\end{array}$ & $\begin{array}{l}1 \\
1 \cdot 0 \\
1 \cdot 2\end{array}$ & $\begin{array}{l}0.5<\mathrm{p}<0.7 \\
(0.6-1 \cdot 7) \\
(0.8-1 \cdot 8)\end{array}$ \\
\hline $\begin{array}{l}\text { Sex partners (past } \\
\quad 0 \\
1 \\
2+\end{array}$ & $\begin{array}{c}5 \text { years) } \\
4 / 210 \\
43 / 636 \\
123 / 1129\end{array}$ & $\begin{array}{r}1.9 \\
6 \cdot 8 \\
10 \cdot 9\end{array}$ & $\begin{array}{l}0 \cdot 4 \\
1 \\
1 \cdot 6\end{array}$ & $\begin{array}{l}0 \cdot 4 \\
1 \\
1 \cdot 7\end{array}$ & $\begin{array}{l}(0 \cdot 1-1 \cdot 2) \\
\mathrm{p}<0 \cdot 001 \dagger \\
(1 \cdot 2-2 \cdot 4)\end{array}$ & $\begin{array}{c}5 / 223 \\
147 / 1400 \\
64 / 500\end{array}$ & $\begin{array}{r}2 \cdot 2 \\
10 \cdot 5 \\
12 \cdot 8\end{array}$ & $\begin{array}{l}0 \cdot 2 \\
1 \\
1 \cdot 2\end{array}$ & $\begin{array}{l}0 \cdot 2 \\
1 \\
1 \cdot 2\end{array}$ & $\begin{array}{l}(0.1-0.6) \\
\mathrm{p}<0.02 \dagger \\
(0.8-1.6)\end{array}$ \\
\hline $\begin{array}{l}\text { Travel to Mwanza } \\
\text { No } \\
\text { Yes }\end{array}$ & $\begin{array}{l}\text { town (past } \\
113 / 1306 \\
56 / 677\end{array}$ & $\begin{array}{l}\text { ars) } \\
8 \cdot 7 \\
8 \cdot 3\end{array}$ & $\begin{array}{l}1 \\
0.9\end{array}$ & $\begin{array}{l}1 \\
0 \cdot 7\end{array}$ & $\begin{array}{l}p=0.16 \\
(0.5-1 \cdot 1)\end{array}$ & $\begin{array}{c}153 / 1690 \\
63 / 454\end{array}$ & $\begin{array}{r}9 \cdot 1 \\
13 \cdot 9\end{array}$ & $\frac{1}{2 \cdot 0}$ & $\begin{array}{l}1 \\
1 \cdot 6\end{array}$ & $\begin{array}{l}\mathrm{p}<0.01 \\
(1.1-2.4)\end{array}$ \\
\hline $\begin{array}{l}\text { Travel outside reg } \\
\text { No } \\
\text { Yes }\end{array}$ & $\begin{array}{l}\text { on (past } 2 y \\
122 / 1403 \\
48 / 595\end{array}$ & $\begin{array}{l}8 \cdot 7 \\
8 \cdot 1\end{array}$ & $\begin{array}{l}1 \\
0 \cdot 8\end{array}$ & $\begin{array}{l}1 \\
0 \cdot 8\end{array}$ & $\begin{array}{l}\mathrm{p}=0.27 \\
(0.6-1 \cdot 2)\end{array}$ & $\begin{array}{c}191 / 1823 \\
25 / 337\end{array}$ & $\begin{array}{r}10 \cdot 5 \\
7 \cdot 4\end{array}$ & $\begin{array}{l}1 \\
0 \cdot 6\end{array}$ & $\begin{array}{l}1 \\
0.5\end{array}$ & $\begin{array}{l}\mathrm{p}=0.01 \\
(0.3-0.9)\end{array}$ \\
\hline $\begin{array}{l}\text { Circumcised } \\
\text { No } \\
\text { Yes }\end{array}$ & $\begin{array}{c}125 / 1354 \\
45 / 642\end{array}$ & $\begin{array}{l}9 \cdot 2 \\
7 \cdot 0\end{array}$ & $\begin{array}{l}1 \\
0 \cdot 6\end{array}$ & $\begin{array}{l}1 \\
0 \cdot 6\end{array}$ & $\begin{array}{l}\mathrm{p}=0.01 \\
(0.4-0.9)\end{array}$ & & & & & \\
\hline
\end{tabular}

*Odds ratio adjusted for age group and residence stratum

†Odds ratio adjusted for age group, residence stratum, marital status, sex partners and travel to Mwanza town.

†Test-for-trend. 
school pupils aged 15 or more. Similar ORs were found in women, but there were fewer women in the non-farming groups and the differences in prevalence were not statistically significant. Men who were separated, divorced or widowed (OR $=1 \cdot 7, \mathrm{CI}: 1 \cdot 1-2 \cdot 7)$ had a higher prevalence of syphilis than married men living with their spouses, while single men had a lower prevalence $(O R=0.4$, CI: 0.3-0.9). Differences among women were less marked, particularly after adjustment for confounders, and were not statistically significant.

In both sexes, syphilis prevalence increased significantly with the reported number of sexual partners during the past five years (test-for-trend gives Males: $p<0.001$; Females: $p<0.02$ ). Men reporting two or more partners had a significantly higher prevalence $(\mathrm{OR}=1 \cdot 7, \mathrm{CI}: 1 \cdot 2-2 \cdot 4)$ than those reporting one partner. In women there was little difference between those reporting one or more than one partner $(O R=1 \cdot 2$, CI: $0 \cdot 8-1 \cdot 6)$, the significant trend resulting mainly from a lower prevalence among women reporting no partners $(O R=0.2$, CI: $0 \cdot 1-0 \cdot 6$ ).

Women (but not men) who had travelled to Mwanza town during the previous two years had an increased prevalence of syphilis (OR $=1 \cdot 6$, CI: $1 \cdot 1-2 \cdot 4)$, while travel outside the region was associated with a reduced prevalence in women $(\mathrm{OR}=0.5, \mathrm{CI}$ : $0.3-0.9$ ).

Among males, prevalence was significantly lower in those who had been circumcised $(\mathrm{OR}=0 \cdot 6, \mathrm{CI}: 0 \cdot 4-0 \cdot 9)$.

Risk factors for GUS and. GD

Overall proportions of respondents reporting at least one episode of genital discharge, now or during the past year, were $6.8 \%$ of men and $4.4 \%$ of women. ${ }^{9}$ Corresponding proportions for genital ulceration were $3.6 \%$ and $2.0 \%$. There was little difference in reported incidence between the three residential strata. Incidences remained fairly stable between ages 15 and 44 years, but were significantly lower in those aged 45 or more (combined incidence of GDS and/or GUS: age 15-44: $7 \cdot 8 \%$; age $45+: 3.5 \%$; $<<0.001$ ).

Incidences of GDS and GUS are analysed according to possible risk factors in tables 2 and 3 respectively. The following associations were identified by this analysis.

The risk of GDS was significantly higher among men with four or more years of school education ( $\mathrm{OR}=2 \cdot 0, \mathrm{CI}: 1 \cdot 2-3 \cdot 6)$. GUS was also more common among these men $(\mathrm{OR}=1 \cdot 6, \mathrm{CI}: 0 \cdot 7-3 \cdot 6)$, although this association was not statistically significant. No such associations were seen among women. Men who were separated, divorced or widowed reported an increased incidence of GDS(OR $=2 \cdot 2, \quad \mathrm{CI}: 1 \cdot 3-3 \cdot 7)$, and a similar but non-significant association was observed for GUS (OR $=1 \cdot 7, \mathrm{CI}: 0 \cdot 8-3 \cdot 5)$. No increased risk was observed among women who were separated, divorced or widowed.

Incidence increased significantly with reported number of sexual partners during the past five years, for both GDS (test-for-trend gives Males: $p<0.001$; Females: $p<0.005$ ) and GUS (Males: $p<0.001$; Females: $\mathrm{p}<0.02)$. Men and women reporting more than one partner were consistently at higher risk of GDS and GUS than those reporting one partner, with lower risks in those reporting no partners.

Incidences of GDS and GUS were higher among men who had travelled to Mwanza town during the past two years (GDS: $\mathrm{OR}=1 \cdot 4$, CI: $0 \cdot 9-2 \cdot 2 ;$ GUS: $\mathrm{OR}=1 \cdot 7$, CI: $0 \cdot 9-3 \cdot 2$ ), although these associations

Table 2 Risk factors for reported genital discharge now or during past year in Mwanza Region

\begin{tabular}{|c|c|c|c|c|c|c|c|c|c|c|}
\hline \multirow[b]{2}{*}{ Risk factor } & \multicolumn{5}{|l|}{ MALES } & \multicolumn{5}{|c|}{ FEMALES } \\
\hline & $+v e / t o t$ & $\%$ & $O R^{*}$ & ORt & $(95 \% C I)$ & $+v e / t o t$ & $\%$ & $O R^{*}$ & ORt & $(95 \% C I)$ \\
\hline $\begin{array}{l}\text { Education } \\
<4 \text { years } \\
\geq 4 \text { years }\end{array}$ & $\begin{array}{c}17 / 470 \\
118 / 1537\end{array}$ & $\begin{array}{l}3 \cdot 6 \\
7 \cdot 7\end{array}$ & $\begin{array}{l}1 \\
2 \cdot 0\end{array}$ & $\begin{array}{l}1 \\
2 \cdot 0\end{array}$ & $\begin{array}{l}\mathrm{p}=0.02 \\
(1 \cdot 2-3.6)\end{array}$ & $\begin{array}{l}43 / 1004 \\
47 / 1157\end{array}$ & $\begin{array}{l}4 \cdot 3 \\
4 \cdot 1\end{array}$ & $\begin{array}{l}1 \\
0.9\end{array}$ & $\begin{array}{l}1 \\
0.9\end{array}$ & $\begin{array}{l}p=0.72 \\
(0.6-1.5)\end{array}$ \\
\hline $\begin{array}{l}\text { Occupation } \\
\text { Farmer } \\
\text { Emp'd/Busin's } \\
\text { Other }\end{array}$ & $\begin{array}{c}91 / 1346 \\
41 / 520 \\
3 / 140\end{array}$ & $\begin{array}{l}6 \cdot 8 \\
7 \cdot 9 \\
2 \cdot 1\end{array}$ & $\begin{array}{l}1 \\
1 \cdot 2 \\
0 \cdot 3\end{array}$ & $\begin{array}{l}1 \\
1 \cdot 1 \\
0 \cdot 3\end{array}$ & $\begin{array}{l}0.05<p<0.1 \\
(0 \cdot 7-1 \cdot 7) \\
(0 \cdot 1-1 \cdot 1)\end{array}$ & $\begin{array}{l}84 / 1971 \\
4 / 139 \\
2 / 53\end{array}$ & $\begin{array}{l}4 \cdot 3 \\
2 \cdot 9 \\
3 \cdot 8\end{array}$ & $\begin{array}{l}1 \\
0 \cdot 6 \\
1 \cdot 0\end{array}$ & $\begin{array}{l}1 \\
0 \cdot 6 \\
1 \cdot 7\end{array}$ & $\begin{array}{l}0 \cdot 2<\mathrm{p}<0 \cdot 3 \\
(0 \cdot 2-1 \cdot 8) \\
(1 \cdot 1-2 \cdot 7)\end{array}$ \\
\hline $\begin{array}{l}\text { Marital status } \\
\text { Married } \\
\text { Single } \\
\text { Sep/Wid/Div }\end{array}$ & $\begin{array}{l}72 / 1192 \\
40 / 617 \\
22 / 181\end{array}$ & $\begin{array}{r}6 \cdot 0 \\
6.5 \\
12 \cdot 2\end{array}$ & $\begin{array}{l}1 \\
0 \cdot 8 \\
2 \cdot 0\end{array}$ & $\begin{array}{l}1 \\
1 \cdot 1 \\
2 \cdot 2\end{array}$ & $\begin{array}{l}0.02<p<0.05 \\
(0.6-1.9) \\
(1.3-3.7)\end{array}$ & $\begin{array}{l}67 / 1412 \\
121 / 379 \\
11 / 358\end{array}$ & $\begin{array}{l}4 \cdot 7 \\
3 \cdot 2 \\
3 \cdot 1\end{array}$ & $\begin{array}{l}1 \\
0 \cdot 7 \\
0 \cdot 7\end{array}$ & $\begin{array}{l}1 \\
1 \cdot 1 \\
0 \cdot 6\end{array}$ & $\begin{array}{l}0.1<\mathrm{p}<0.2 \\
(0.5-2 \cdot 3) \\
(0.3-1 \cdot 3)\end{array}$ \\
\hline $\begin{array}{l}\text { Sex partners (past } \\
0 \\
1 \\
2+\end{array}$ & $\begin{array}{c}5 \text { years) } \\
7 / 211 \\
32 / 1441 \\
94 / 1134\end{array}$ & $\begin{array}{l}3 \cdot 3 \\
5 \cdot 0 \\
8 \cdot 3\end{array}$ & $\begin{array}{l}0 \cdot 4 \\
1 \\
1 \cdot 9\end{array}$ & $\begin{array}{l}0.5 \\
1 \\
1 \cdot 8\end{array}$ & $\begin{array}{l}(0 \cdot 2-1 \cdot 3) \\
\mathrm{p}<0.001 \neq \\
(1 \cdot 1-2 \cdot 7)\end{array}$ & $\begin{array}{l}3 / 223 \\
56 / 1404 \\
30 / 500\end{array}$ & $\begin{array}{l}1 \cdot 3 \\
4 \cdot 0 \\
6 \cdot 0\end{array}$ & $\begin{array}{l}0.4 \\
1 \\
1 \cdot 6\end{array}$ & $\begin{array}{l}0 \cdot 3 \\
1 \\
1 \cdot 7\end{array}$ & $\begin{array}{l}(0 \cdot 1-1 \cdot 1) \\
\mathrm{p}<0 \cdot 005 \dagger \\
(1 \cdot 1-2 \cdot 7)\end{array}$ \\
\hline $\begin{array}{l}\text { Travel to Mwanza } \\
\text { No } \\
\text { Yes }\end{array}$ & $\begin{array}{c}\text { town (past } \\
79 / 1994 \\
54 / 678\end{array}$ & $\begin{array}{r}4.0 \\
8.0\end{array}$ & $\begin{array}{l}1 \\
1.5\end{array}$ & $\begin{array}{l}1 \\
1 \cdot 4\end{array}$ & $\begin{array}{l}p=0.15 \\
(0.9-2 \cdot 2)\end{array}$ & $\begin{array}{l}67 / 1694 \\
23 / 454\end{array}$ & $\begin{array}{l}4 \cdot 0 \\
5 \cdot 1\end{array}$ & $\begin{array}{l}1 \\
1 \cdot 4\end{array}$ & $\begin{array}{l}1 \\
1 \cdot 4\end{array}$ & $\begin{array}{l}p=0.22 \\
(0.8-2.4)\end{array}$ \\
\hline $\begin{array}{l}\text { Travel outside regi } \\
\text { No } \\
\text { Yes }\end{array}$ & $\begin{array}{l}\text { on (past } 2 \text { y } \\
88 / 1411 \\
47 / 598\end{array}$ & $\begin{array}{l}6.2 \\
7.9\end{array}$ & $\begin{array}{l}1 \\
1 \cdot 2\end{array}$ & $\begin{array}{l}1 \\
1 \cdot 1\end{array}$ & $\begin{array}{l}p=0.73 \\
(0.7-1.6)\end{array}$ & $\begin{array}{l}77 / 1827 \\
13 / 337\end{array}$ & $\begin{array}{l}4 \cdot 2 \\
3 \cdot 9\end{array}$ & $\begin{array}{l}1 \\
0.9\end{array}$ & $\begin{array}{l}1 \\
0.9\end{array}$ & $\begin{array}{l}p=0.85 \\
(0.5-1.8)\end{array}$ \\
\hline $\begin{array}{l}\text { Circumcised } \\
\text { No } \\
\text { Yes }\end{array}$ & $\begin{array}{l}77 / 1356 \\
58 / 646\end{array}$ & $\begin{array}{l}5 \cdot 7 \\
9 \cdot 0\end{array}$ & $\begin{array}{l}1 \\
1 \cdot 7\end{array}$ & $\begin{array}{l}1 \\
1.8\end{array}$ & $\begin{array}{l}p=0.01 \\
(1.2-2.6)\end{array}$ & & & & & \\
\hline
\end{tabular}

*Odds ratio adjusted for age group and residence stratum.

†Odds ratio adjusted for age group, residence stratum, marital status, sex partners and travel to Mwanza town. tTest-for-trend. 
Table 3 Risk factors for reported genital ulcer now or during past year in Mwanza Region

\begin{tabular}{|c|c|c|c|c|c|c|c|c|c|c|}
\hline \multirow[b]{2}{*}{ Risk factor } & \multicolumn{5}{|l|}{$M A L E S$} & \multicolumn{5}{|c|}{ FEMALES } \\
\hline & $+v e / t o t$ & $\%$ & $O R^{*}$ & OR† & $(95 \% C I)$ & $+v e / t o t$ & $\%$ & $O R^{*}$ & OR† & $(95 \% C I)$ \\
\hline $\begin{array}{l}\text { Education } \\
\quad<4 \text { years } \\
\geq 4 \text { years }\end{array}$ & $\begin{array}{l}9 / 470 \\
61 / 1537\end{array}$ & $\begin{array}{l}1.9 \\
4.0\end{array}$ & $\begin{array}{l}1 \\
1 \cdot 8\end{array}$ & $\begin{array}{l}1 \\
1.6\end{array}$ & $\begin{array}{l}p=0.24 \\
(0.7-3.6)\end{array}$ & $\begin{array}{l}23 / 1004 \\
24 / 1157\end{array}$ & $\begin{array}{l}2 \cdot 3 \\
2 \cdot 1\end{array}$ & $\begin{array}{l}1 \\
0 \cdot 7\end{array}$ & $\begin{array}{l}1 \\
0.6\end{array}$ & $\begin{array}{l}\mathrm{p}=0.17 \\
(0 \cdot 3-1 \cdot 2)\end{array}$ \\
\hline $\begin{array}{l}\text { Occupation } \\
\text { Farmer } \\
\text { Emp'd/Busin's } \\
\text { Other }\end{array}$ & $\begin{array}{c}48 / 1346 \\
19 / 520 \\
3 / 140\end{array}$ & $\begin{array}{l}3 \cdot 6 \\
3 \cdot 7 \\
2 \cdot 1\end{array}$ & $\begin{array}{l}1 \\
1 \cdot 3 \\
0 \cdot 6\end{array}$ & $\begin{array}{l}1 \\
1 \cdot 0 \\
0 \cdot 7\end{array}$ & $\begin{array}{l}p>0.9 \\
(0.5-1.9) \\
(0 \cdot 2-2 \cdot 6)\end{array}$ & $\begin{array}{l}45 / 1971 \\
2 / 139 \\
0 / 53\end{array}$ & $\begin{array}{l}2 \cdot 3 \\
1 \cdot 4 \\
0 \cdot 0\end{array}$ & $\begin{array}{l}1 \\
0 \cdot 6 \\
0 \cdot 0\end{array}$ & $\begin{array}{l}1 \\
0 \cdot 5 \\
0 \cdot 0\end{array}$ & $\begin{array}{l}\mathrm{p}=0.36 \\
(0.1-2 \cdot 2)\end{array}$ \\
\hline $\begin{array}{l}\text { Marital status } \\
\text { Married } \\
\text { Single } \\
\text { Sep/Wid/Div }\end{array}$ & $\begin{array}{c}38 / 1192 \\
20 / 617 \\
9 / 181\end{array}$ & $\begin{array}{l}3 \cdot 2 \\
3 \cdot 2 \\
5 \cdot 0\end{array}$ & $\begin{array}{l}1 \\
0 \cdot 6 \\
1 \cdot 4\end{array}$ & $\begin{array}{l}1 \\
0 \cdot 7 \\
1 \cdot 7\end{array}$ & $\begin{array}{l}0.2<\mathrm{p}<0.3 \\
(0.4-1.6) \\
(0.8-3.5)\end{array}$ & $\begin{array}{c}30 / 1412 \\
10 / 379 \\
7 / 358\end{array}$ & $\begin{array}{l}2 \cdot 1 \\
2 \cdot 6 \\
2 \cdot 0\end{array}$ & $\begin{array}{l}1 \\
1 \cdot 1 \\
1 \cdot 0\end{array}$ & $\begin{array}{l}1 \\
1.9 \\
1 \cdot 0\end{array}$ & $\begin{array}{l}0.3<p<0.5 \\
(0 \cdot 8-4 \cdot 6) \\
(0 \cdot 4-2 \cdot 4)\end{array}$ \\
\hline $\begin{array}{l}\text { Sex partners (past } \\
0 \\
1 \\
2+\end{array}$ & $\begin{array}{c}5 \text { years) } \\
3 / 211 \\
13 / 641 \\
53 / 1134\end{array}$ & $\begin{array}{l}1 \cdot 4 \\
2 \cdot 0 \\
4 \cdot 7\end{array}$ & $\begin{array}{l}0 \cdot 5 \\
1 \\
2 \cdot 7\end{array}$ & $\begin{array}{l}0.7 \\
1 \\
3 \cdot 0\end{array}$ & $\begin{array}{l}(0 \cdot 2-3 \cdot 0) \\
\mathrm{p}<0 \cdot 001 \ddagger \\
(1 \cdot 5-6 \cdot 0)\end{array}$ & $\begin{array}{l}11 / 223 \\
30 / 1404 \\
15 / 500\end{array}$ & $\begin{array}{l}4 \cdot 9 \\
2 \cdot 1 \\
3 \cdot 0\end{array}$ & $\begin{array}{l}0 \cdot 2 \\
1 \\
1 \cdot 3\end{array}$ & $\begin{array}{l}0 \cdot 1 \\
1 \\
1 \cdot 4\end{array}$ & $\begin{array}{l}(0.0-0.8) \\
p<0.02 \dagger \\
(0.7-2.6)\end{array}$ \\
\hline $\begin{array}{l}\text { Travel to Mwanza } \\
\text { No } \\
\text { Yes }\end{array}$ & $\begin{array}{l}\text { town (pas } \\
36 / 1994 \\
32 / 678\end{array}$ & $\begin{array}{l}\text { ars) } \\
1 \cdot 8 \\
4 \cdot 7\end{array}$ & $\begin{array}{l}1 \\
2 \cdot 0\end{array}$ & $\begin{array}{l}1 \\
1 \cdot 7\end{array}$ & $\begin{array}{l}\mathrm{p}=0.10 \\
(0.9-3 \cdot 2)\end{array}$ & $\begin{array}{l}46 / 1694 \\
12 / 454\end{array}$ & $\begin{array}{l}2 \cdot 7 \\
2 \cdot 6\end{array}$ & $\begin{array}{l}1 \\
1 \cdot 1\end{array}$ & $\begin{array}{l}1 \\
1.0\end{array}$ & $\begin{array}{l}\mathrm{p}=0.95 \\
(0.5-2 \cdot 2)\end{array}$ \\
\hline $\begin{array}{l}\text { Travel outside reg } \\
\text { No } \\
\text { Yes }\end{array}$ & $\begin{array}{l}\text { on (past } 2 \\
58 / 1411 \\
12 / 598\end{array}$ & $\begin{array}{l}4 \cdot 1 \\
2 \cdot 0\end{array}$ & $\begin{array}{l}1 \\
0 \cdot 5\end{array}$ & $\begin{array}{l}1 \\
0 \cdot 3\end{array}$ & $\begin{array}{l}\mathrm{p}=0.001 \\
(0.2-0.6)\end{array}$ & $\begin{array}{c}40 / 1827 \\
7 / 337\end{array}$ & $\begin{array}{l}2 \cdot 2 \\
2 \cdot 1\end{array}$ & $\begin{array}{l}1 \\
0.9\end{array}$ & $\begin{array}{l}1 \\
0 \cdot 8\end{array}$ & $\begin{array}{l}\mathrm{p}=0.61 \\
(0.3-1.9)\end{array}$ \\
\hline $\begin{array}{l}\text { Circumcised } \\
\text { No } \\
\text { Yes }\end{array}$ & $\begin{array}{l}52 / 1356 \\
18 / 646\end{array}$ & $\begin{array}{l}3 \cdot 8 \\
2 \cdot 8\end{array}$ & $\begin{array}{l}1 \\
0.7\end{array}$ & $\begin{array}{l}1 \\
0.7\end{array}$ & $\begin{array}{l}\mathrm{p}=0.26 \\
(0.4-1.3)\end{array}$ & & & & & \\
\hline
\end{tabular}

*Odds ratio adjusted for age group and residence stratum

HOdds ratio adjusted for age group, residence stratum, marital status, sex partners and travel to Mwanza town.

fTest-for-trend.

were not statistically significant. Men who had travelled outside the region had a significantly reduced risk of GUS (OR $=0.3, \mathrm{CI}$ : $0 \cdot 2-0 \cdot 6)$. No significant associations between travel and incidence were observed among women.

Male circumcision was associated with a significantly increased risk of GDS (OR = 1.8; CI: $1 \cdot 2-2 \cdot 6$ ), but showed no association with GUS.

\section{Health seeking behaviour}

Of those reporting that they had ever had genital discharge, $98 \%$ of men and $90 \%$ of women had sought treatment. Of those seeking treatment, $68 \%$ of men and $61 \%$ of women sought treatment from official health facilities (dispensaries, health centres or hospitals), $21 \%$ of men and $34 \%$ of women from traditional healers, and $17 \%$ of men and $6 \%$ of women from other sources. These percentages add to more than $100 \%$, because a few subjects reported seeking treatment from more than one source. Traditional treatment was sought more frequently in rural villages (Males: 24\%; Females: $38 \%$ ) than in urban (Males: $10 \%$; Females: $24 \%$ ) or roadside (Males: $18 \%$; Females: $24 \%$ ) strata.

Of those reporting that they had ever had genital ulcers, $97 \%$ of men and $90 \%$ of women had sought treatment. Of these, $71 \%$ of men and $61 \%$ of women sought treatment from official health facilities, $19 \%$ of men and $32 \%$ of women from traditional healers, and $13 \%$ of men and $8 \%$ of women from other sources. Again, traditional treatment was sought more frequently in rural villages (Males: 22\%; Females: $37 \%$ ) than in urban (Males: $11 \%$; Females: $18 \%$ ) or roadside (Males: 14\%; Females: 23\%) strata.

\section{Discussion}

To our knowledge, there has been only one previous population-based study of bacterial STDs and their risk factors in a rural African community. Arya et al reported in 1973 on the clinical, cultural and demographic aspects of gonorrhoea in the Teso district of Uganda, which was known to have an extremely high rate of female infertility. ${ }^{10}$ The point prevalence of urethral discharge among males was $32 / 270(12 \%)$ and $55 \%$ of males reported ever having a discharge (compared with $2 \cdot 6 \%$ and $27 \cdot 3 \%$ among rural males in the present study). The Ugandan study attempted genital examination of all eligible subjects, and this may partly account for the higher prevalences recorded. Of urethral discharges in males, $75 \%$ were due to gonorrhoea. In contrast to the present study, the highest prevalence of gonorrhoea (24\%) was observed in the 50-59 year age group; the only other risk factor identified was living with more than one wife. Single men had a significantly lower prevalence, the prevalence among separated, widowed or divorced men was the same as among men living with one wife, and educational status did not affect prevalence.

The prevalence of gonorrhoea among women in the Ugandan study was $18 \%$ $(54 / 295)$. In contrast to men, the highest prevalence $(28 \%)$ was observed in the youngest age group. ${ }^{15-19}$ Separated, widowed or divorced women had a higher prevalence than married or single women, and women involved in polygamous marriages had a higher prevalence than those whose husbands had never had more than one wife.

There are thus certain risk factors in common between our study and that in Uganda, and others which differ. It is likely that risk 
factors for STDs vary between populations, as a result of differences in the social and cultural context, and there is a need for further studies in other parts of Africa.

Although only limited information on sexual behaviour was collected in the present study, and of unknown accuracy, a clear association was observed in men between syphilis and STD syndromes and the reported number of sexual partners during the past five years. Significant associations with number of sexual partners were observed also among women, but these resulted mainly from a lower risk in those reporting no partners. Only for GDS was there a significant difference in risk between those reporting one partner and two or more partners. Assuming that interview responses on sexual partners were equally accurate in males and females, a possible interpretation would be that STD infections in women are acquired mainly from their spouses, the data obtained from this survey suggesting that many of the women with one partner have spouses reporting more than one partner.

Men who were separated, divorced or widowed had a higher prevalence of syphilis and more frequently reported GDS and GUS than men who were married and living with their spouses, or single. This relationship may be explained by a higher frequency of unstable sexual partnerships among separated, divorced or widowed men, in which case the associations would be expected to weaken after adjustment for number of sexual partners. In fact, the associations persisted, but this may reflect a lack of precision in the data on number of partners. Women who were separated, divorced or widowed did not appear to be at an increased risk of STDs, which may imply that the frequency of unstable partnerships in this group is less than among men.

Conflicting results were observed for the relationship between STDs and education. School education of four years or more was associated with a higher risk of GDS or GUS in men, but with a lower prevalence of syphilis in women. This observation is in keeping with studies of risk factors for HIV infection. Several studies have suggested that higher socioeconomic status confers an increased risk among males, ${ }^{11} 12$ possibly as a result of greater disposable cash incomes. In females, this is not the case, and Over and Piot have drawn attention to an inverse correlation between the proportion of females enrolled in secondary school and the HIV prevalence in the general population of Central African cities. ${ }^{7}$ The lower prevalence of syphilis among educated women may partly result from such women receiving treatment more promptly.

Travelling to the regional centre (Mwanza town) was associated with a significantly higher prevalence of syphilis in women, and showed the same tendency for GUS and GDS in men although the latter associations were not statistically significant. Visiting the town may be associated with risk behaviour, but it also seems possible that some individuals travelled to town because they were seeking treatment for an STD.

Results for travelling outside Mwanza Region showed a reduced risk of syphilis in women, and GUS in men. These observations are difficult to interpret in the absence of information about the destination of travel, which is not available. In the same study, no increased risk of HIV infection was found in those travelling outside the region. ${ }^{8}$

Male circumcision was associated with a reduced prevalence of syphilis, but an increased incidence of GDS. Male circumcision is much more prevalent (about $60 \%$ ) in Mwanza town than in rural areas, but the associations remained after adjustment for residence stratum. In the same study, no association was observed between male circumcision and HIV infection. ${ }^{8}$ It is difficult to dissociate any biological effect of circumcision on the risk of STD from cultural and behavioural factors associated with circumcision. A possible explanation for our findings is that circumcised men may have a more "urban" lifestyle and have more sexual partners than the uncircumcised, leading to a higher incidence of GDS, but that circumcision exerts a biologically protective effect against syphilis and HIV infection, as has been suggested by others. ${ }^{13} 14$

The high proportion of both males and females with symptomatic STDs seeking treatment in the official health sector is encouraging. This confirms the conclusions of a survey which we undertook in 1991 of STD cases treated in rural health facilities in the region, from which we estimated that up to $80 \%$ of subjects with symptomatic genital ulceration or discharge were treated in the official sector. ${ }^{9}$ However, we are aware that training of health care staff in the recognition and management of STDs is deficient, and that the treatment provided may be ineffective. A regionwide programme for the improved detection and management of STDs is currently being implemented, and its impact on the prevalence and incidence of STDs will be evaluated.

An additional problem is that a high proportion of females with STDs are known to be asymptomatic, and we have recently documented a high prevalence of asymptomatic STDs among males attending hospital in Mwanza with symptoms unrelated to the genital tract. ${ }^{15}$ The identification and treatment of these asymptomatic individuals is perhaps the greatest challenge facing STD control programmes in developing countries.

We thank the Principal Secretary, Ministry of Health and the Director-General of the National Institute for Medical Research for permission to carry out and publish the results of this study. The support of regional, district, ward and village government and party officials is gratefully acknowledged. We are particularly grateful for the support and hospitality of the people included in the survey. This study was supported by the EC AIDS Task Force, the Netherlands Minister for the EC AIDS Task Force, the Netherlands Minister for Development Cooperation, the Overseas Development Trust. 
1 De Schryver A, Meheus A. Epidemiology of sexually transmitted diseases: the global picture. Bull World Health Organiz 1990;68:639-54.

2 Schulz K, Cates W, O'Masra PR. Pregnancy loss, infant death, and suffering: legacy of syphilis and gonorrhoea in Africa. Genitourin Med 1987;62:320-5.

3 Muir DG, Belsey MA. Pelvic inflammatory disease and its consequences in the developing world. Am $\mathcal{f}$ Obstet Gynecol 1980;138:913-28.

4 Pepin J, Plummer FA, Brunham RC, Piot P, Cameron DW, Ronald AR. The interaction of HIV and other sexually transmitted diseases: an opportunity for intervention. AIDS 1989;3:3-9.

5 Cameron DW, Simonsen JN, D'Costa LJ, et al. Female to male transmission of human immunodeficiency virus type 1: risk factors for seroconversion in men. Lancet 1989;ii:403-7.

6 Plummer FA, Simonsen JN, Cameron DW, et al. Cofactors in male-female transmission of human immunodeficiency virus type 1. F Infect Dis 1991;163: 233-9.

7 Over M, Piot P. HIV infection and sexually transmitted diseases. Forthcoming in: Jamison DT and Mosley WH (eds): Disease Control Prionities in Developing Countries. New York: Oxford University Press for the Wountries. Nank

8 Barongo LR, Borgdorff MW, Mosha FF, et al, The epidemiology of HIV-1 infection in urban areas, roadside settlements and rural villages in Mwanza Region, Tanzania. AIDS 1992;6:1521-8.

9 Mosha F, Nicoll A, Barongo L, et al. A population-based study of syphilis and sexually transmitted disease syndromes in North-Western Tanzania. 1. Prevalence and incidence. Genitourin Med 1993;00:415-20.

10 Arya OP, Nsanzumuhire H, Taber SR. Clinical, cultural and demographic aspects of gonorrhoea in a community in Uganda. Bull World Health Organ 1973;49:587-95.

11 Melbye M, Nselesani EK, Bayley A. Evidence for heterosexual transmission and clinical manifestations of human immunodeficiency virus and related conditions in Lusaka, Zambia. Lancet 1986;ii:1113-5.

12 Bugingo G, Ntilivamundi A, Nzaramba D, et al. Etude sur la seropositivite liée à infection au virus de l'immunodeficience humaine au Rwanda. Revue Medicale Rwandaise 1988;20:37-42.

13 Moses S, Bradley JE, Nagelkerke NJD, Ronald AR, Ndinya-Achola JD, Plummer FA. Geographical patterns of male circumcision practices in Africa: association with HIV seroprevalence. Int $\mathcal{F}$ Epidemiol 1990;19: 693-7.

14 Bongaarts J, Reining $P$, Way $P$, Conant $F$. The relationship between male circumcision and HIV infection in ship between male circumsision and

15 Mayaud $P$, Changalucha J, Grosskurth $H$, et al. The value of urine specimens in screening for male urethritis and of urine specimens in screening for male urethritis and its aetiold 